Article

\title{
New Unsymmetrically Benzene-Fused Bis (Tetrathiafulvalene): Synthesis, Characterization, Electrochemical Properties and Electrical Conductivity of Their Materials
}

Tahar Abbaz $^{1{ }^{1 *} \text {, Amel Bendjeddou }}{ }^{1}$, Abdelkrim Gouasmia ${ }^{2}$, Didier Villemin ${ }^{3}$ and Takashi Shirahata ${ }^{4}$

1 Laboratory of Aquatic and Terrestrial Ecosystems, Organic and Bioorganic Chemistry Group, University of Mohammed Cherif Mesaadia, Souk Ahras 41000, Algeria;

E-Mail: bendjeddouamel@gmail.com

2 Laboratory of Organic Materials and Heterochemistry, University of Tebessa, Constantine Road, Tebessa 12000, Algeria; E-Mail: akgouasmia@hotmail.com

3 Laboratory of Molecular and Thio-Organic Chemistry, UMR CNRS 6507, INC3M, FR 3038, Labex EMC3, ENSICAEN \& University of Caen, Caen 14050, France;

E-Mail: didier.villemin@ensicaen.fr

4 Department of Applied Chemistry, Graduate School of Science and Engineering, Ehine University, 3 Bunkyo-cho, Matsuyama, Ehine 790-8577, Japan; E-Mail: shirahata.takashi.mj@ehime-u.ac.jp

* Author to whom correspondence should be addressed; E-Mail: tahar.abbaz@yahoo.com; Tel.: +213-792-272-719; Fax: +213-30-952-935.

Received: 19 February 2014; in revised form: 7 March 2014 / Accepted: 10 March 2014 / Published: 17 March 2014

\begin{abstract}
The synthesis of new unsymmetrically benzene-fused bis (tetrathiafulvalene) has been carried out by a cross-coupling reaction of the respective 4,5-dialkyl-1,3-dithiole2-selenone 6-9 with 2-(4-( $p$-nitrophenyl)-1,3-dithiole-2-ylidene)-1,3,5,7-tetrathia-sindacene-6-one 5 prepared by olefination of 4-( $p$-nitrophenyl)-1,3-dithiole-2-selenone 3 and 1,3,5,7-tetrathia-s-indacene-2,6-dione 4 . The conversion of the nitro moiety $\mathbf{1 0 a}-\mathbf{d}$ to amino $\mathbf{1 1 a}-\mathbf{d}$ then dibenzylamine $\mathbf{1 2 a}-\mathbf{d}$ groups respectively used reduction and alkylation methods. The electron donor ability of these new compounds has been measured by cyclic voltammetry $(\mathrm{CV})$ technique. Charge transfer complexes with tetracyanoquino-dimethane (TCNQ) were prepared by chemical redox reactions. The complexes have been proven to give conducting materials.
\end{abstract}


Keywords: conductivity; redox potentials; organic materials; tetrathiafulvalenes

\section{Introduction}

The tetrathiafulvalene (TTF) molecule has attracted great interest since the early 1970's, when scientists saw its high electrical conductivity in a chloride salt and its metallic behaviour in the charge transfer complex, tetrathiafulvalene-tetracyanoquinodimethane (TTF-TCNQ). Recently, new applications of TTF and its derivatives in supramolecular [1-3] and materials chemistry [4-6] have been developed by TTF block building more flexible than was previously appreciated.

TTF derivatives now play a significant role as redox sites in different areas of supramolecular chemistry. Some applications call for their use as cations sensors [7-9] as a $\pi$-electron donor for non-linear optical systems [10-12], heterocycles [13-15], integrated into polymeric [16-18] and dendritic systems [19] and used as a component for molecular electronic devices [20-22].

Among the wide variety of chemical modifications performed on the TTF skeleton, the synthesis of highly extended and sulfur rich systems has recently received particular attention [23-25]. Fused aromatic rings (benzene, naphthalene, pyrazine, or quinoxaline rings) [26-28] onto the TTF skeleton are known to be an attractive electron-donor molecule which can provide a highly conductive charge transfer complex owing to its highly extended $p$-conjugate part.

As a development of our previous work [29-34] and taking into account the above, we decided to design and realize the synthesis of novel unsymmetrically benzene-fused bis (tetrathiafulvalene) containing nitrophenyl, aminophenyl or dibenzylaminophenyl units.

We report in this work the synthesis the electrochemical properties of such compounds and finally we also prepared their charge transfer complexes and measured their electrical conductivity.

\section{Results and Discussion}

As shown in Scheme 1, commercially available 2-( $p$-nitrophenyl)-2-oxoethyl 1-piperidinecarbodithioate 1 was cyclized by the concentrated sulfuric acid at $0{ }^{\circ} \mathrm{C}$. The resulting hydrogenosulfate was converted to 4-( $p$-nitrophenyl)-1,3-dithiole-2-ylidenepiperidinium hexafluorophosphate 2 immediately by addition of hexafluorophosphoric acid. After recrystallization, the desired product was obtained in $65 \%$ yield. The treatment of compound $\mathbf{2}$ with sodium hydrogen selenide, prepared in situ from selenium and sodium borohydride in ethanol at low temperature, followed by an aqueous work up, afford after filtration and purification over silica gel chromatography the desired product 4-( $p$-nitrophenyl)-1,3-dithiole-2selenone 3 in $93 \%$ yield.

Scheme 1. Synthetic route for the preparation of 4-( $p$-nitrophenyl)-1,3-dithiole-2-selenone 3.

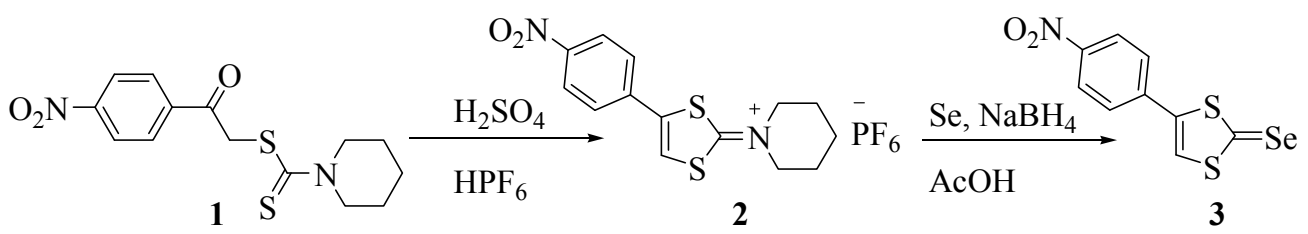


Scheme 2 exhibits the synthetic routes for the preparation of compounds 10a-d. The condensation via cross coupling method [35] of The 4-( $p$-nitrophenyl)-1,3-dithiole-2-selenone 3 with 1,3,5,7-tetrathia-sindacene-2,6-dione 4 [28], in toluene at reflux in the presence of triethyl phosphite under nitrogen, leads to the formation of the desired 2-(4-( $p$-nitrophenyl)-1,3-dithiole-2-ylidene)-1,3,5,7-tetrathia-sindacene-6-one 5 in moderate yield (45\%) after column chromatography. The coupling reaction between various selenones 6-9 [36-39] and 1,3,5,7-tetrathia-s-indacene-6-one 5 with a large excess of triethyl phosphite while refluxing in toluene successfully afforded the $p$-nitrophenyl benzene-fused bis tetrathiafulvalenes $\mathbf{1 0 a}-\mathbf{d}$ in $36 \%, 42 \%, 44 \%$ and $32 \%$ yields, respectively.

Scheme 2. Route for the preparation of $p$-nitrophenyl benzene-fused bis tetrathiafulvalenes 10a-d.

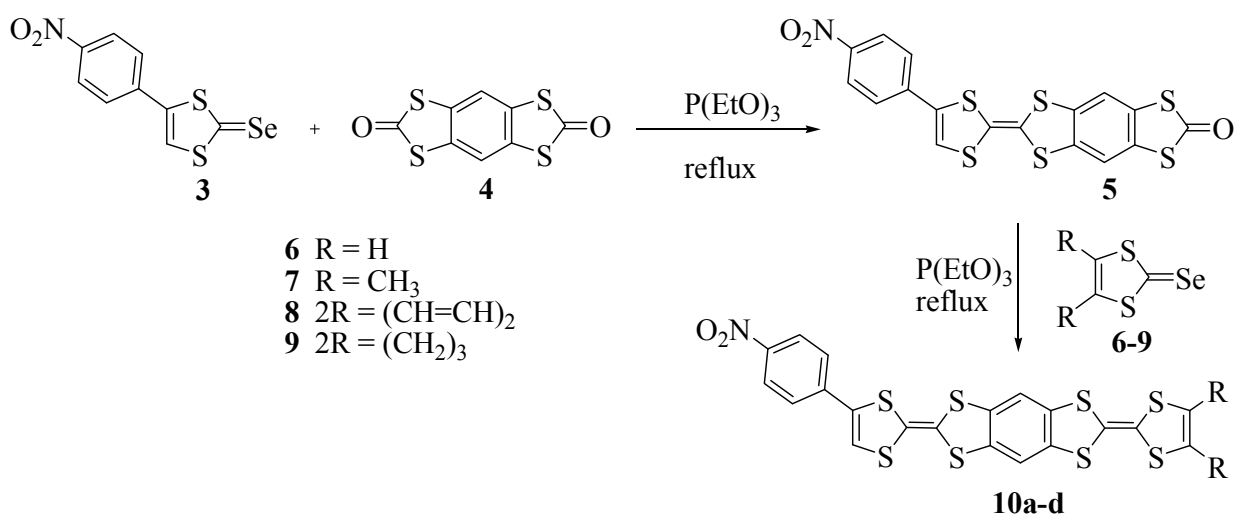

In previous work [29] we have described the access to alkylated aminophenyl bis-TTFs from nitrophenyl bis-TTFs. In Scheme 3, the nitro group of $p$-nitrophenyl benzene-fused bis tetrathiafulvalenes $\mathbf{1 0 a}-\mathbf{d}$ was reduced at reflux in the presence of tin and hydrochloric acid into an amino group in ethanol. The $p$-aminophenyl benzene-fused bis tetrathiafulvalene 11a-d derivatives were obtained after purification by column chromatography in 74\%,77\%, 79\% and 71\% yields, respectively. Then, their alkylation was effected by treatment with $\mathrm{K}_{2} \mathrm{CO}_{3}$ (2 equiv.) and with 2 equivalents of benzyl bromide in DMF at reflux, the dibenzylaminophenyl benzene-fused bis TTFs 12a-d were obtained in $87 \%, 95 \%, 93 \%$ and $85 \%$ yields, respectively, after purification by column chromatography.

Scheme 3. Synthetic route for the preparation of dibenzylaminophenyl benzene-fused bis TTFs 12a-d.
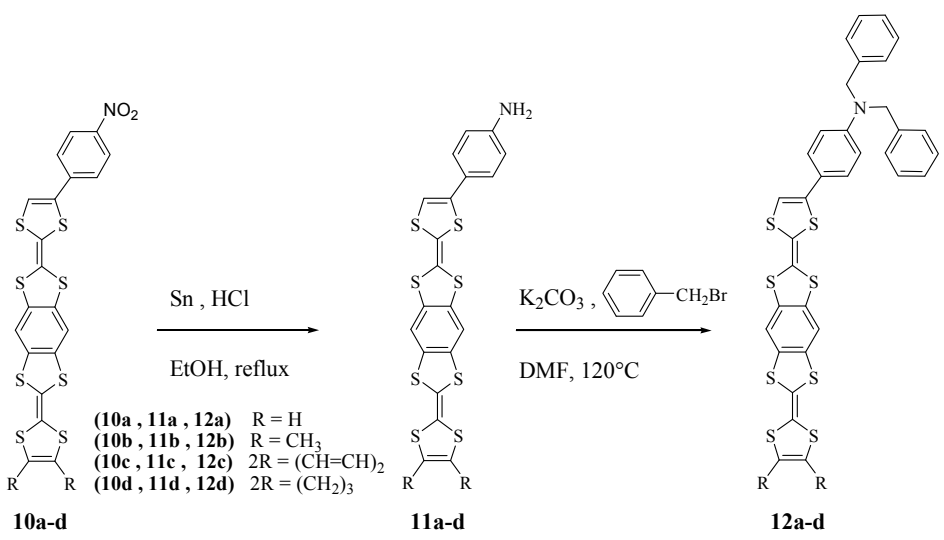
In the ${ }^{1} \mathrm{H}$ NMR spectra the series of $p$-nitrophenyl benzene-fused bis tetrathiafulvalene 10a-d exhibited two doublets around 7.42-7.44 and 8.08-8.10 ppm for the nitrophenyl protons. The series of $p$-aminophenyl benzene-fused bis tetrathiafulvalene 11a-d revealed the presence of amino group protons signals as broad band around 3.48-3.75 ppm and the aminophenyl protons showed two doublets around $6.40-6.42$ and 6.98-7.00 ppm. Thus, the series of $p$-dibenzylaminophenyl benzene-fused bis tetrathiafulvalene 12a-d showed the absence of the amino group proton signals and the presence of benzylamine protons as singlet around 4.65-4.67 ppm and a multiplet around 7.13-7.28 ppm.

Mass spectrometry analysis validated the structure of the examined derivatives. In all compounds, fragmentation peaks confirmed the structure of the analyzed molecules.

\subsection{Electrochemical Studies}

The redox properties of these new functional unsymmetrically benzene-fused bis TTFs were studied in solution by cyclic voltammetry $(\mathrm{CV})$ and by square wave voltammetry (SQW). Measurements were performed under nitrogen at room temperature using a glassy carbon working electrode, a Pt counter electrode and a standard calomel electrode (SCE) as reference, with tetrabutylammonium perchlorate $\left(n-\mathrm{Bu}_{4} \mathrm{NClO}_{4}, 0.1 \mathrm{M}\right)$ in dry acetonitrile, as supporting electrolyte. A scan rate of $100 \mathrm{mV} \cdot \mathrm{s}^{-1}$ was used. The CV measurements showed reversible redox waves for all the compounds studied and the corresponding oxidation potentials $E_{\mathrm{ox}}$ were determined by the SQW technique. The results are summarized in Table 1.

Table 1. Potential of unsymmetrically benzene-fused bis tetrathiafulvalenes $\mathbf{1 0 a}-\mathbf{1 2 d}$.

\begin{tabular}{|c|c|c|c|c|}
\hline Donor & $E^{1}{ }_{0 x}(m V)$ & $E_{0 x}^{2}(\mathrm{mV})$ & $E^{3}{ }_{0 x}(m V)$ & $\Delta E_{\mathrm{ox}}(\mathrm{mV})$ \\
\hline $10 \mathrm{a}$ & 461 & 530 & 873 & 412 \\
\hline $10 \mathrm{~b}$ & 459 & 528 & 870 & 411 \\
\hline $10 \mathrm{c}$ & 456 & 524 & 866 & 410 \\
\hline 10d & 457 & 525 & 867 & 410 \\
\hline $11 \mathrm{a}$ & 438 & 507 & 845 & 407 \\
\hline $11 b$ & 436 & 504 & 842 & 406 \\
\hline $11 \mathrm{c}$ & 433 & 499 & 836 & 403 \\
\hline 11d & 434 & 501 & 838 & 404 \\
\hline $12 \mathrm{a}$ & 446 & 525 & 864 & 418 \\
\hline $12 b$ & 445 & 522 & 861 & 416 \\
\hline $12 \mathrm{c}$ & 441 & 522 & 854 & 413 \\
\hline 12d & 443 & 523 & 858 & 415 \\
\hline
\end{tabular}

In Figure 1, we can clearly see three oxidation peaks with respectively a 1, 1 and 2 electron process. The real distinction of the two first oxidation waves is clearly due to the difference between the effect donor and the effect attractor of the substituents carried by the two units TTF, which also visible by cyclic voltammetry.

The oxidation potentials of compounds $\mathbf{1 2 a}-\mathbf{d}$ are slightly higher than that of compounds $\mathbf{1 1 a}-\mathbf{d}$, on the other hand, the compounds $\mathbf{1 0 a}-\mathbf{d}$ are slightly higher than that of compounds $\mathbf{1 2 a}-\mathbf{d}$. This should be attributable to the electron-donating capabilities of these new compounds by the presence of the $p$-nitrophenyl, $p$-aminophenyl and $p$-dibenzylaminophenyl groups linked to the donor core. 
Figure 1. Voltammogram of benzene-fused bis TTF 10a, 11a and 12a.

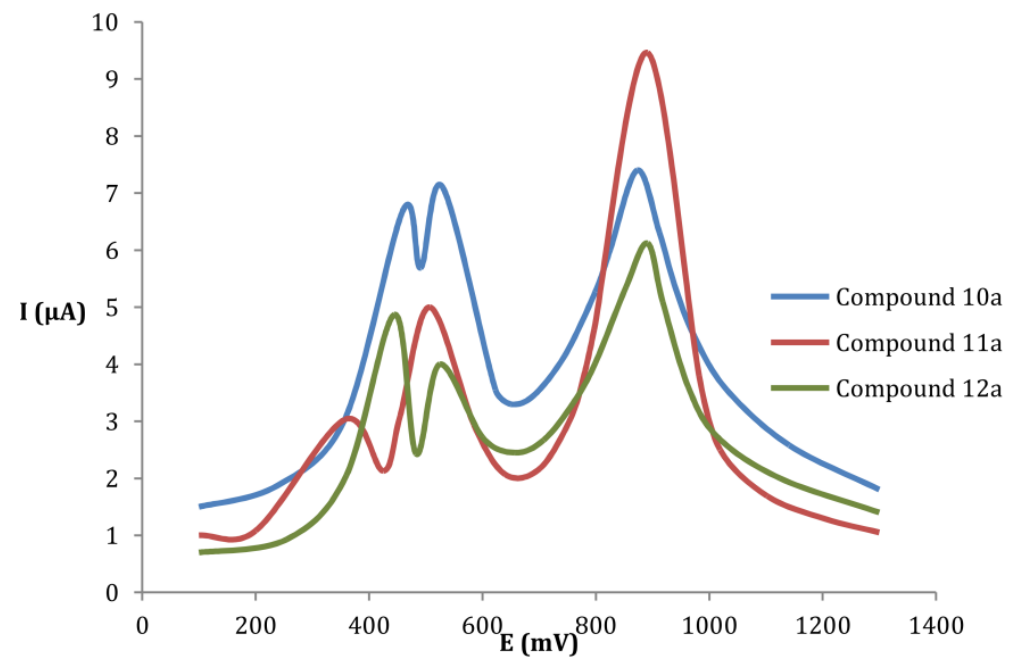

In the same series, the presence of alkyl groups on the TTF skeleton enriches the electron density and facilitates the oxidation of the donor, which it is noted for compound 10d and 10b compared with 10a, while the presence of the aromatic group extends the conjugated system and improves the electron density; it was clearly visible for compound 10c which showed the lowest oxidation potential in this series. Similar results were observed for the other series of $p$-aminophenyl and p-dibenzylaminophenyl groups.

\subsection{Theoretical Calculation}

The energy of HOMO of different products 10a to 12d was computed using DFT calculation in the Table 2. The levels of HOMO of compound $\mathbf{1 2 b}(-4.507 \mathrm{eV})$ and $\mathbf{1 2 d}(-4.516 \mathrm{eV})$ show that these compounds are the better donating molecule for the formation of TTF-TCNQ complexes.

Table 2. Energy level $(\mathrm{eV})$ of the molecular orbitals for products $\mathbf{1 0 a}-\mathbf{1 2 d}$.

\begin{tabular}{cccccccc}
\hline Compound & LUMO + 2 & LUMO + 1 & LUMO & HOMO & HOMO - 1 & HOMO - 2 & HOMO - 3 \\
\hline 10a & -1.184 & -1.460 & -2.684 & -4.901 & -5.249 & -6.459 & -6.580 \\
10b & -1.121 & -1.414 & -2.664 & -4.807 & -5.189 & -6.330 & -6.509 \\
$\mathbf{1 0 c}$ & -1.218 & -1.471 & -2.688 & -4.977 & -5.304 & -6.460 & -6.558 \\
$\mathbf{1 0 d}$ & -1.114 & -1.421 & -2.670 & -4.782 & -5.192 & -6.289 & -6.532 \\
$\mathbf{1 1 a}$ & -0.758 & -0.912 & -1.137 & -4.597 & -4.889 & -5.636 & -6.300 \\
$\mathbf{1 1 b}$ & -0.726 & -0.868 & -1.073 & -4.542 & -4.805 & -5.602 & -6.187 \\
$\mathbf{1 1 c}$ & -0.774 & -0.940 & -1.166 & -4.631 & -4.989 & -5.651 & -6.308 \\
$\mathbf{1 1 d}$ & -0.732 & -0.863 & -1.078 & -4.538 & -4.786 & -5.604 & -6.151 \\
$\mathbf{1 2 a}$ & -0.747 & -0.900 & -1.127 & -4.564 & -4.865 & -5.416 & -6.238 \\
$\mathbf{1 2 b}$ & -0.709 & -0.859 & -1.064 & -4.507 & -4.782 & -5.385 & -6.124 \\
$\mathbf{1 2 c}$ & -0.764 & -0.927 & -1.157 & -4.596 & -4.963 & -5.432 & -6.253 \\
$\mathbf{1 2 d}$ & -0.723 & -0.851 & -1.064 & -4.516 & -4.763 & -5.389 & -6.088 \\
\hline
\end{tabular}


Figure 2 shows that the nature of the alkyl groups has little influence on the level of the HOMO and in consequence on the potential of oxidation, which can be also found in Table 1. Three groups of compound can be obtained: $10 \mathbf{a}-\mathbf{d}, \mathbf{1 1} \mathbf{a}-\mathbf{d}$ and $\mathbf{1 2 a}-\mathbf{d}$.

Figure 2. Levels of HOMO and LOMO of compounds 10a-d.

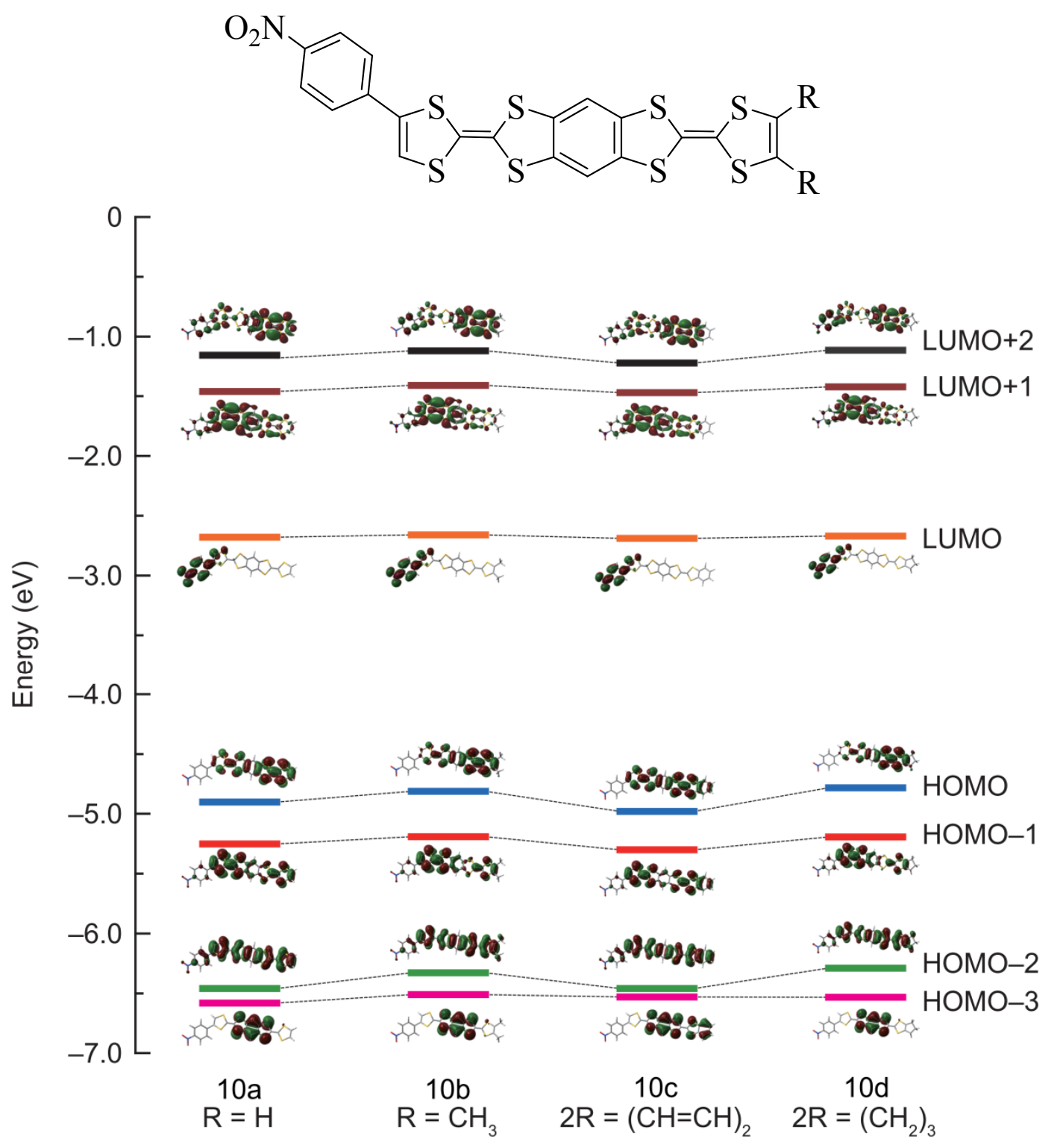

In Figure 3 the levels of the HOMO of $\mathbf{1 0 d}$ and $\mathbf{1 2 d}$ shows that compound $\mathbf{1 2 d}$ is more oxidable than 10d, however in the case of 11d the amine group can take part in oxidation which makes difficult a correlation between the level of the HOMO of $\mathbf{1 1 d}$ and its facility of oxidation. 
Figure 3. Levels of HOMO and LOMO of compounds $\mathbf{1 0 d}, \mathbf{1 1 d}$ and $\mathbf{1 2 d .}$

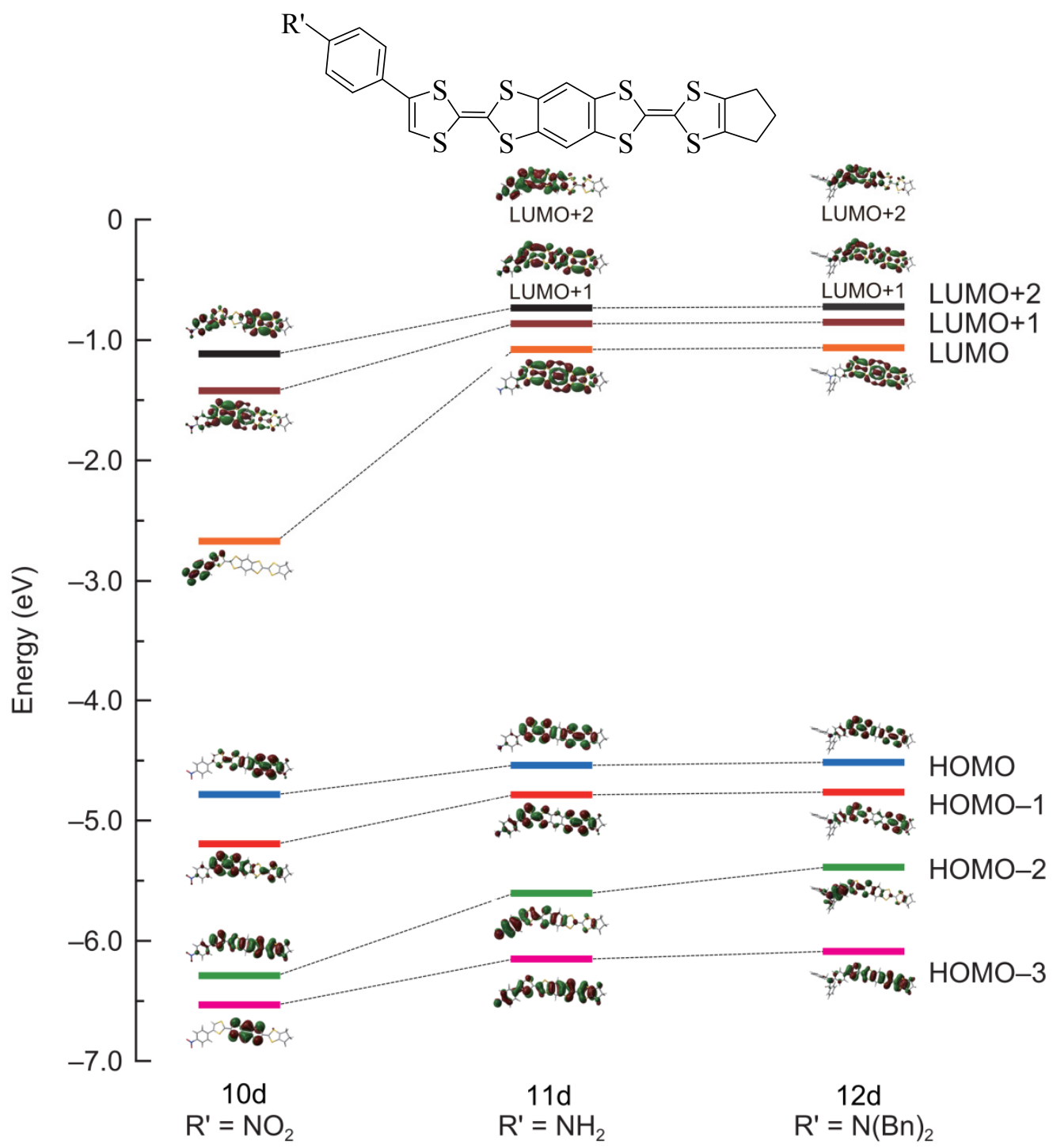

\subsection{Preparation and Electrical Conductivity of Charge Transfer Complexes}

Charge transfer complexes (CTC) are a special case where metallic-like conductivites are obtained from essentially non-metallic, organic molecules. A CTC is formed by the interaction of an electron donor (D) and an electron acceptor (A). Electron donors are compounds with low ionization potential, while electron acceptors are compounds with high electron affinity. The donor and acceptor are bound together by an electrostatic attraction, not a chemical bond. Partial electron transfer between the donor molecule and the acceptor molecule generates this electrostatic attraction.

In our study, all compounds 10a-12d formed charge transfer complexes with TCNQ (tetracyano-p-quinodimethane) used as an electron acceptor (A) [40-42]. The solids were isolated after cooling the hot acetonitrile solution obtained by mixing equimolar amounts of the donor (D) and of TCNQ (A). Most of the materials were obtained as powders with various colors.

The room temperature conductivity of these solids was measured by using a two probe technique on compressed pellets. The results obtained are summarized in Table 3. 
Table 3. Melting points and electrical conductivity of charge transfer complexes.

\begin{tabular}{ccc}
\hline Complex & M.P $\left({ }^{\circ} \mathbf{C}\right)$ & $\boldsymbol{\sigma R T}\left(\mathbf{S ~ c m}^{-1}\right)$ \\
\hline 10a-TCNQ & 276 & $9.2 \times 10^{-2}$ \\
10b-TCNQ & 281 & $4.8 \times 10^{-1}$ \\
10c-TCNQ & 289 & $5.3 \times 10^{-1}$ \\
10d-TCNQ & 294 & $8.7 \times 10^{-1}$ \\
11a-TCNQ & 227 & $2.5 \times 10^{-2}$ \\
11b-TCNQ & 231 & $1.7 \times 10^{-2}$ \\
11c-TCNQ & 237 & $8.3 \times 10^{-1}$ \\
11d-TCNQ & 240 & $7.6 \times 10^{-1}$ \\
12a-TCNQ & 258 & $8.7 \times 10^{-6}$ \\
12b-TCNQ & 263 & $5.3 \times 10^{-5}$ \\
12c-TCNQ & 267 & $4.2 \times 10^{-4}$ \\
12d-TCNQ & 272 & $1.8 \times 10^{-4}$ \\
\hline
\end{tabular}

For this family of materials, only CTC from 10a-TCNQ to 11d-TCNQ resulting from $p$-nitrophenyl benzene-fused bis tetrathiafulvalenes and $p$-aminophenyl benzene-fused bis tetrathiafulvalenes, can be classified in the area of conductors. In fact, they have a conductivity measured on powder compressed pellets of $4.8 \times 10^{-1}$ to $9.2 \times 10^{-2} \mathrm{~S} \mathrm{~cm}^{-1}$, which allows conductivity ten times greater on single crystal.

Other, CTC resulting from $p$-dibenzylaminophenyl benzene-fused bis tetrathiafulvalenes from 12a-TCNQ to 12d-TCNQ can be classified in the category of semi-conductors materials with conductivities from $10^{-4}$ to $10^{-6} \mathrm{~S} \mathrm{~cm}^{-1}$. This can be due to a structural disorder and/or a full charge transfer of an electron for each molecule.

\section{Experimental Section}

\subsection{General}

NMR spectra were recorded on a WP 400-NMR instrument (Bruker BioSpin GmbH, Silberstreifen 4, 76287 Rheinstetten, Germany). FAB mass spectra were recorded on a JOEL JMS-DX 300 spectrometer (JEOL Europe, Planet II, Gebouw B., Leuvensestreenweg 542, B-1930 Zaventem, Belgium). Uncorrected melting points were measured on a 510 Buchi apparatus (BÜCHI Labortechnik AG, Meierseggstrasse 40, 9230 Flawil, Schweiz). Cyclic voltammetry measurements were carried out on a PAR-273 potentiostat/galvanostat (Alltest Instruments, Inc. 500 Central Ave. Farmingdale, NJ, USA). All computations were performed with the Gaussian 09 program package (Gaussian, Inc. 340 Quinnipiac St, Bldg 40, Wallingford, CT, USA) [43] using the 6-31G(d,p) basis set [44]. Density functional theory (DFT) calculations were carried out using a B3LYP method (public field method) [45-47]. All solvents were dried by standard methods and all commercial reagents used without purification. All reactions were performed under an inert atmosphere of nitrogen. 


\subsection{Synthesis and Characterization of 4-(p-Nitrophenyl)-1,3-dithiole-2-ylidenepiperidinium Hexafluorophosphate 2}

2-(p-Nitrophenyl)-2-oxoethyl 1-piperidinecarbodithioate 1 (19.44 g, $0.06 \mathrm{~mol})$ was added drop wise to a stirred solution of concentrated sulfuric acid $(45 \mathrm{~mL})$ at $0{ }^{\circ} \mathrm{C}$. After the reaction mixture was allowed to warm to ambient temperature, cold water $(150 \mathrm{~mL})$ was added and the mixture was filtered. The residual solution was cooled to $0{ }^{\circ} \mathrm{C}$ and hexafluorophosphoric acid $(6.5 \mathrm{~mL}, 0.06 \mathrm{~mol})$ was added drop wise over $2 \mathrm{~min}$, yellow suspension was observed in the solution, and the reaction was allowed to reach room temperature. The reaction was extracted with $\mathrm{CH}_{2} \mathrm{CI}_{2}(3 \times 100 \mathrm{~mL})$. The organic extracts were combined and washed with water $(3 \times 100 \mathrm{~mL})$ and dried $\left(\mathrm{MgSO}_{4}\right)$. The solvent was removed under reduced pressure. The crude product was recrystallised from ethanol to give $2(65 \%)$ as beige solid. M.p.: $173{ }^{\circ} \mathrm{C} .{ }^{1} \mathrm{H}$ NMR (400 MHz, $\left.\mathrm{CDCl}_{3}, \delta, \mathrm{ppm}\right): 1.78$ (m, 6H, Py-H), 3.28 (m, 4H, Py-H), 7.45 (s, 1H, CH=C-S), 7.73 (d, $J=8.70 \mathrm{~Hz}, 2 \mathrm{H}$, nitrophenyl- $H$ ), 8.17 (d, $J=8.70 \mathrm{~Hz}$, $2 \mathrm{H}$, nitrophenyl- $H$ ). MS (NOBA, FAB $>0$ ): $453[\mathrm{M}+\mathrm{H}]^{+}$. Anal. calcd. for $\mathrm{C}_{14} \mathrm{H}_{15} \mathrm{~S}_{2} \mathrm{~N}_{2} \mathrm{O}_{2} \mathrm{PF}_{6}: \mathrm{C}$, 37.17; H, 3.34; S, 14.17; found: C, 36.87; H, 3.04; S, 14.47 .

\subsection{Synthesis and Characterization of 4-(p-Nitrophenyl)-1,3-dithiole-2-selenone 3}

Black powdered selenium $(2.8 \mathrm{~g}, 35.37 \mathrm{mmol})$ was added in one portion to a solution of sodium borohydride $(7.7 \mathrm{~g}, 70.74 \mathrm{mmol})$ in ethanol $(40 \mathrm{~mL})$ with magnetic stirring at $0{ }^{\circ} \mathrm{C}$ under argon. A vigorous reaction with considerable foaming immediately occurred and the selenium was consumed in less than $30 \mathrm{~min}$. The virtually colorless solution of NaHSe, which resulted was ready for use without further treatment. After cooling of the solution acetic acid $(2 \mathrm{~mL}, 35.37 \mathrm{mmol})$ and 4 - $p$-nitrophenyl -1,3-dithiole-2-ylidenepiperidinium hexafluorophosphate (15.98 g, $35.37 \mathrm{mmol}$ ) were added and the reaction mixture was allowed to stand at room temperature for $c a .2 \mathrm{~h}$. The ethanol was diluted to $100 \%$ with deoxygenated ice water and the red solid was filtered, washed with water, dried under vacuum and chromatographed (silica gel, $\mathrm{CHCl}_{3}$ ). Recrystallization of the product from heptane gave 3 (9.94 g, 93\% yield) as red orange crystals. M.p.: $146{ }^{\circ} \mathrm{C}$. TLC:Rf $=0.90\left(\mathrm{CH}_{2} \mathrm{Cl}_{2}\right) .{ }^{1} \mathrm{H}$ NMR (400 MHz, $\left.\mathrm{CDCl}_{3}, \delta, \mathrm{ppm}\right): 6.94(\mathrm{~s}, 1 \mathrm{H}, \mathrm{C}=\mathrm{C} H), 7.57$ (d, $J=8.65 \mathrm{~Hz}, 2 \mathrm{H}$, nitrophenyl- $H$ ), 8.17 (d, $J=8.65 \mathrm{~Hz}, 2 \mathrm{H}$, nitrophenyl- $H$ ); $\mathrm{MS}$ (NOBA, FAB $>0$ ): $303[\mathrm{M}+\mathrm{H}]^{+}$. Anal. calcd. for $\mathrm{C}_{9} \mathrm{H}_{5} \mathrm{~S}_{2} \mathrm{SeNO}_{2}$ : C, 35.76; H, 1.66; S, 21.21; found: C, 35.46; H, 1.46; S, 21.51.

\subsection{Synthesis and Characterization of 2-(4-(p-Nitrophenyl)-1,3-dithiole-2-ylidene)-1,3,5,7-tetrathia-s-} indacene-6-one 5

Under a nitrogen atmosphere, $25 \mathrm{~mL}$ of freshly distilled triethyl phosphite was added to the mixture of 4-(p-nitrophenyl)-1,3-dithiole-2-selenone 3 (1 g, $3.31 \mathrm{mmol})$ and 1,3,5,7-tetrathia-s-indacene-2,6dione 4 (1 equiv.). The resulting mixture was heated with an oil bath up to $110{ }^{\circ} \mathrm{C}$ and stirred for a further $4 \mathrm{~h}$. The solvent was then removed under reduced pressure. Compound 5 was obtained by column chromatography of the residue (silica gel, eluting with dichloromethane and petroleum ether 2:1) in $45 \%$ yield. Light yellow powder, M.p.: $132{ }^{\circ} \mathrm{C}$. TLC: $\mathrm{Rf}=0.83\left(\mathrm{CH}_{2} \mathrm{Cl}_{2}\right) .{ }^{1} \mathrm{H} \mathrm{NMR}\left(400 \mathrm{MHz}, \mathrm{CDCl}_{3}\right.$, $\delta$, ppm): $6.83(\mathrm{~s}, 1 \mathrm{H}, \mathrm{C}=\mathrm{C} H), 7.11(\mathrm{~s}, 2 \mathrm{H}$, benzene-fused- $H), 7.45(\mathrm{~d}, J=8.87 \mathrm{~Hz}, 2 \mathrm{H}$, nitrophenyl- $H)$, 
$8.10\left(\mathrm{~d}, J=8.87 \mathrm{~Hz}, 2 \mathrm{H}\right.$, nitrophenyl- $H$ ); MS (NOBA, FAB $>0$ ): $466[\mathrm{M}+\mathrm{H}]^{+}$. Anal. calcd. for $\mathrm{C}_{17} \mathrm{H}_{7} \mathrm{~S}_{6} \mathrm{NO}_{3}$ : C, 43.85; H, 1.51; S, 41.31; found: C, 44.00; H, 1.71; S, 41.01.

\subsection{Synthesis and Characterization of p-Nitrophenyl Benzene-Fused Bis Tetrathiafulvalene 10a-d}

Compounds $\mathbf{1 0 a}-\mathbf{d}$ were synthesized by employing the same experimental process as $\mathbf{5}$ from 1 equiv. of 5 and 1 equiv. of various selenones $6-9$.

p-Nitrophenyl benzene-fused bis tetrathiafulvalene 10a: Dark blue powder. Yield: $36 \%$. M.p.: $168{ }^{\circ} \mathrm{C}$. TLC: $\mathrm{Rf}=0.70\left(\mathrm{CH}_{2} \mathrm{Cl}_{2} /\right.$ petroleum ether, 2:1). ${ }^{1} \mathrm{H} \mathrm{NMR}\left(400 \mathrm{MHz}, \mathrm{CDCl}_{3}, \delta, \mathrm{ppm}\right): 6.37(\mathrm{~s}, 2 \mathrm{H}$, $\mathrm{C} H=\mathrm{C} H), 6.84(\mathrm{~s}, 1 \mathrm{H}, \mathrm{C}=\mathrm{C} H), 6.95(\mathrm{~s}, 2 \mathrm{H}$, benzene-fused- $H), 7.44(\mathrm{~d}, J=8.88 \mathrm{~Hz}, 2 \mathrm{H}$, nitrophenyl- $H)$, $8.10(\mathrm{~d}, J=8.88 \mathrm{~Hz}, 2 \mathrm{H}$, nitrophenyl- $H$ ). MS (NOBA, FAB $>0): 552[\mathrm{M}+\mathrm{H}]^{+}$. Anal. calcd. for $\mathrm{C}_{20} \mathrm{H}_{9} \mathrm{~S}_{8} \mathrm{NO}_{2}$ : C, 43.53; H, 1.64; S, 46.48; found: C, 43.73; H, 1.74; S, 46.18.

$p$-Nitrophenyl benzene-fused bis tetrathiafulvalene 10b: Midnight blue powder. Yield: 42\%. M.p.: $175{ }^{\circ} \mathrm{C}$. TLC: $\mathrm{Rf}=0.65\left(\mathrm{CH}_{2} \mathrm{Cl}_{2} /\right.$ petroleum ether, 2:1). ${ }^{1} \mathrm{H}$ NMR $\left(400 \mathrm{MHz}, \mathrm{CDCl}_{3}, \delta, \mathrm{ppm}\right): 1.95$ $\left(\mathrm{s}, 6 \mathrm{H}, \mathrm{CH}_{3}\right), 6.82(\mathrm{~s}, 1 \mathrm{H}, \mathrm{C}=\mathrm{CH}), 6.91(\mathrm{~s}, 2 \mathrm{H}$, benzene-fused- $H), 7.42(\mathrm{~d}, J=8.86 \mathrm{~Hz}, 2 \mathrm{H}$, nitrophenyl- $H$ ), $8.08(\mathrm{~d}, J=8.86 \mathrm{~Hz}, 2 \mathrm{H}$, nitrophenyl- $H)$. MS (NOBA, FAB $>0): 580[\mathrm{M}+\mathrm{H}]^{+}$. Anal. calcd. for $\mathrm{C}_{22} \mathrm{H}_{13} \mathrm{~S}_{8} \mathrm{NO}_{2}$ : C, 45.56; $\mathrm{H}, 2.25$; $\mathrm{S}, 44.23$; found: $\mathrm{C}, 45.86 ; \mathrm{H}, 2.55 ; \mathrm{S}, 43.93$.

$p$-Nitrophenyl benzene-fused bis tetrathiafulvalene 10c: Indigo powder. Yield: 44\%. M.p.: $184{ }^{\circ} \mathrm{C}$. TLC: $\mathrm{Rf}=0.54\left(\mathrm{CH}_{2} \mathrm{Cl}_{2} /\right.$ petroleum ether, $\left.2: 1\right) .{ }^{1} \mathrm{H} \mathrm{NMR}\left(400 \mathrm{MHz}, \mathrm{CDCl}_{3}, \delta, \mathrm{ppm}\right): 6.83(\mathrm{~s}, 1 \mathrm{H}$, $\mathrm{C}=\mathrm{C} H), 6.93(\mathrm{~s}, 2 \mathrm{H}$, benzene-fused- $H), 7.00-7.30(\mathrm{~m}, 4 \mathrm{H}$, benzene- $H), 7.43(\mathrm{~d}, J=9.00 \mathrm{~Hz}, 2 \mathrm{H}$, nitrophenyl- $H$ ), $8.10\left(\mathrm{~d}, J=9.00 \mathrm{~Hz}, 2 \mathrm{H}\right.$, nitrophenyl- $H$ ). MS (NOBA, FAB > 0): $602[\mathrm{M}+\mathrm{H}]^{+}$. Anal. calcd. for $\mathrm{C}_{24} \mathrm{H}_{11} \mathrm{~S}_{8} \mathrm{NO}_{2}$ : C, 47.89; H, 1.84; S, 42.62; found: C, 48.09; H, 2.04; S, 42.52.

p-Nitrophenyl benzene-fused bis tetrathiafulvalene 10d: Blue violet powder. Yield: $32 \%$. M.p.: $188{ }^{\circ} \mathrm{C}$. TLC: $\mathrm{Rf}=0.58\left(\mathrm{CH}_{2} \mathrm{Cl}_{2}\right.$ /petroleum ether, 2:1). ${ }^{1} \mathrm{H} \mathrm{NMR}\left(400 \mathrm{MHz}, \mathrm{CDCl}_{3}, \delta, \mathrm{ppm}\right): 2.45(\mathrm{q}, J=6.9 \mathrm{~Hz}$, $\left.2 \mathrm{H}, \mathrm{CH}_{2}\right), 2.56\left(\mathrm{t}, J=6.9 \mathrm{~Hz}, 4 \mathrm{H}, 2 \mathrm{CH}_{2}\right), 6.82(\mathrm{~s}, 1 \mathrm{H}, \mathrm{C}=\mathrm{CH}), 6.91$ (s, 2H, benzene-fused- $\left.H\right), 7.42$ (d, $J=8.87 \mathrm{~Hz}, 2 \mathrm{H}$, nitrophenyl- $H$ ), 8.08 (d, $J=8.87 \mathrm{~Hz}, 2 \mathrm{H}$, nitrophenyl- $H$ ). MS (NOBA, FAB $>0$ ): $592[\mathrm{M}+\mathrm{H}]^{+}$. Anal. calcd. for $\mathrm{C}_{23} \mathrm{H}_{13} \mathrm{~S}_{8} \mathrm{NO}_{2}$ : C, 46.67; H, 2.21; S, 43.34; found: C, 46.77; H, 2.31; S, 43.19 .

\subsection{Synthesis and Characterization of p-Aminophenyl Benzene-Fused Bis Tetrathiafulvalene 11a-d}

A stirred mixture of 4- $p$-nitrophenyl benzene-fused bis TTFs derivatives 10a-d (4 mmol), tin $(0.94 \mathrm{~g}, 8 \mathrm{mmol})$, and aqueous solution of $\mathrm{HCl}(35 \%)$ to $(1.8 \mathrm{~mL}, 20 \mathrm{mmol})$ in ethanol $(30 \mathrm{~mL})$ was refluxed for $4 \mathrm{~h}$ under nitrogen. During this time the initial black solution turned light yellow. The solution was then concentrated in vacuo and treated with an aqueous solution $(100 \mathrm{~mL})$ of sodium hydroxide $(0.1 \mathrm{M})$ and extracted with ether. The organic phase was washed with water, dried $\left(\mathrm{MgSO}_{4}\right)$, and concentrated in vacuo. The product was subjected to column chromatography on silica gel $\left(\mathrm{CH}_{2} \mathrm{Cl}_{2}\right)$, affording the expected compounds $11 \mathbf{a}-\mathbf{d}$ as powder.

p-Aminophenyl benzene-fused bis tetrathiafulvalene 11a: Dark orange powder. Yield: 74\%. M.p.: $127^{\circ} \mathrm{C}$. TLC: $\mathrm{Rf}=0.72\left(\mathrm{CH}_{2} \mathrm{Cl}_{2}\right) .{ }^{1} \mathrm{H}$ NMR $\left(400 \mathrm{MHz}, \mathrm{CDCl}_{3}, \delta, \mathrm{ppm}\right): 3.50-3.75\left(\mathrm{br}, 2 \mathrm{H}, \mathrm{NH}_{2}\right), 6.37$ (s, 2H, $\mathrm{CH}=\mathrm{CH}), 6.42(\mathrm{~d}, J=8.48 \mathrm{~Hz}, 2 \mathrm{H}$, aminophenyl- $H), 6.60(\mathrm{~s}, 1 \mathrm{H}, \mathrm{C}=\mathrm{CH}), 6.95(\mathrm{~s}, 2 \mathrm{H}$, benzene-fused- $H$ ), $7.00\left(\mathrm{~d}, J=8.48 \mathrm{~Hz}, 2 \mathrm{H}\right.$, aminophenyl- $H$ ); MS (NOBA, FAB $>0$ ): $522[\mathrm{M}+\mathrm{H}]^{+}$. Anal. calcd. for $\mathrm{C}_{20} \mathrm{H}_{11} \mathrm{~S}_{8} \mathrm{~N}$ : C, 46.03; H, 2.12; S, 49.15; found: C, 46.22; H, 2.27; S, 48.83 . 
p-Aminophenyl benzene-fused bis tetrathiafulvalene 11b: Orange powder. Yield: 77\%. M.p.: $133{ }^{\circ} \mathrm{C}$. TLC: $\mathrm{Rf}=0.67\left(\mathrm{CH}_{2} \mathrm{Cl}_{2}\right) .{ }^{1} \mathrm{H}$ NMR $\left(400 \mathrm{MHz}, \mathrm{CDCl}_{3}, \delta, \mathrm{ppm}\right): 1.95\left(\mathrm{~s}, 6 \mathrm{H}, \mathrm{CH}_{3}\right), 3.48-3.73(\mathrm{br}, 2 \mathrm{H}$, $\left.\mathrm{NH}_{2}\right), 6.40(\mathrm{~d}, J=8.26 \mathrm{~Hz}, 2 \mathrm{H}$, aminophenyl- $H), 6.58(\mathrm{~s}, 1 \mathrm{H}, \mathrm{C}=\mathrm{CH}), 6.91(\mathrm{~s}, 2 \mathrm{H}$, benzene-fused- $H)$, $6.98\left(\mathrm{~d}, J=8.26 \mathrm{~Hz}, 2 \mathrm{H}\right.$, aminophenyl- $H$ ). MS (NOBA, FAB $>0$ ): $550[\mathrm{M}+\mathrm{H}]^{+}$. Anal. calcd. for $\mathrm{C}_{22} \mathrm{H}_{15} \mathrm{~S}_{8} \mathrm{~N}$ : C, 48.05; H, 2.74; S, 46.64; found: C, 48.33; H, 2.97; S, 46.36.

p-Aminophenyl benzene-fused bis tetrathiafulvalene 11c: Coral powder. Yield: $79 \%$. M.p.: $142{ }^{\circ} \mathrm{C}$. TLC: $\mathrm{Rf}=0.56\left(\mathrm{CH}_{2} \mathrm{Cl}_{2}\right) .{ }^{1} \mathrm{H}$ NMR $\left(400 \mathrm{MHz}, \mathrm{CDCl}_{3}, \delta, \mathrm{ppm}\right): 3.59-3.89\left(\mathrm{br}, 2 \mathrm{H}, \mathrm{NH}_{2}\right), 6.59(\mathrm{~s}, 1 \mathrm{H}$, $\mathrm{C}=\mathrm{CH}), 6.41(\mathrm{~d}, J=8.60 \mathrm{~Hz}, 2 \mathrm{H}$, aminophenyl- $H), 6.93(\mathrm{~s}, 2 \mathrm{H}$, benzene-fused- $H), 6.99(\mathrm{~d}, J=8.60 \mathrm{~Hz}$, $2 \mathrm{H}$, aminophenyl- $H$ ), 7.00-7.30 (m, 4H, benzene- $H$ ). MS (NOBA, FAB > 0): $572[\mathrm{M}+\mathrm{H}]^{+}$. Anal. calcd. for $\mathrm{C}_{24} \mathrm{H}_{13} \mathrm{~S}_{8} \mathrm{~N}$ : C, 50.40; H, 2.29; S, 44.85; found: C, 50.70; H, 2.39; S, 45.20.

p-Aminophenyl benzene-fused bis tetrathiafulvalene 11d: Orange red powder. Yield: 71\%. M.p.: $146{ }^{\circ} \mathrm{C}$. TLC: $\mathrm{Rf}=0.60\left(\mathrm{CH}_{2} \mathrm{Cl}_{2}\right) .{ }^{1} \mathrm{H}$ NMR $\left(400 \mathrm{MHz}, \mathrm{CDCl}_{3}, \delta, \mathrm{ppm}\right): 2.45\left(\mathrm{q}, J=6.9 \mathrm{~Hz}, 2 \mathrm{H}, \mathrm{CH}_{2}\right)$, $2.56\left(\mathrm{t}, J=6.9 \mathrm{~Hz}, 4 \mathrm{H}, 2 \mathrm{CH}_{2}\right), 3.48-3.73\left(\mathrm{br}, 2 \mathrm{H}, \mathrm{N} H_{2}\right), 6.40(\mathrm{~d}, J=8.47 \mathrm{~Hz}, 2 \mathrm{H}$, aminophenyl- $H$ ), $6.58(\mathrm{~s}, 1 \mathrm{H}, \mathrm{C}=\mathrm{CH}), 6.91$ (s, $2 \mathrm{H}$, benzene-fused- $H), 6.98(\mathrm{~d}, J=8.47 \mathrm{~Hz}, 2 \mathrm{H}$, aminophenyl- $H$ ). MS (NOBA, FAB > 0): $562[\mathrm{M}+\mathrm{H}]^{+}$. Anal. calcd. for $\mathrm{C}_{23} \mathrm{H}_{15} \mathrm{~S}_{8} \mathrm{~N}$ : C, 49.16; H, 2.69; S, 45.65; found: C, 49.28; H, 2.84; S, 45.46.

\subsection{Synthesis and Characterization of p-Dibenzylaminophenyl Benzene-Fused Bis Tetrathiafulvalene 12a-d}

$\mathrm{K}_{2} \mathrm{CO}_{3}(0.83 \mathrm{~g}, 6 \mathrm{mmol})$ was added to a stirred solution of 4-aminophenyl benzene-fused bis TTF 11a-d $(3 \mathrm{mmol})$ and benzyl bromide $(0.71 \mathrm{~mL}, 6 \mathrm{mmol})$ in dimethylformamide $(30 \mathrm{~mL})$ under nitrogen. The resulting mixture was heated over an oil bath up to $120{ }^{\circ} \mathrm{C}$ and stirred for a further $2 \mathrm{~h}$. The solvent was then removed under reduced pressure. Compound 12a-d was obtained by column chromatography of the residue (silica gel, eluting with dichloromethane).

$p$-Dibenzylaminophenyl benzene-fused bis tetrathiafulvalene 12a: Light yellow powder. Yield: 87\%. M.p.: $195{ }^{\circ} \mathrm{C}$. TLC: Rf $=0.81\left(\mathrm{CH}_{2} \mathrm{Cl}_{2}\right) .{ }^{1} \mathrm{H}$ NMR $\left(400 \mathrm{MHz}, \mathrm{CDCl}_{3}, \delta\right.$, ppm): 4.67 (s, 4H, benzylamine- $\left.\mathrm{CH}_{2}\right), 6.37(\mathrm{~s}, 2 \mathrm{H}, \mathrm{CH}=\mathrm{CH}), 6.54(\mathrm{~s}, 1 \mathrm{H}, \mathrm{C}=\mathrm{CH}), 6.57(\mathrm{~d}, J=8.66 \mathrm{~Hz}, 2 \mathrm{H}$, aminophenyl- $H), 6.95(\mathrm{~s}, 2 \mathrm{H}$, benzene-fused- $H), 7.15-7.25(\mathrm{~m}, 10 \mathrm{H}$, benzylamine- $H), 7.35(\mathrm{~d}$, $J=8.66 \mathrm{~Hz}, 2 \mathrm{H}$, aminophenyl- $H$ ). MS (NOBA, FAB $>0$ ): $702[\mathrm{M}+\mathrm{H}]^{+}$. Anal. Calcd for $\mathrm{C}_{36} \mathrm{H}_{21} \mathrm{~S}_{8} \mathrm{~N}$ : C, 58.16; H, 3.30; S, 36.53; found: C, 58.03; H, 3.18; S, 36.68 .

p-Dibenzylaminophenyl benzene-fused bis tetrathiafulvalene 12b: Wheat powder. Yield: 95\%. M.p.: $208{ }^{\circ} \mathrm{C}$. TLC: $\mathrm{Rf}=0.76\left(\mathrm{CH}_{2} \mathrm{Cl}_{2}\right) .{ }^{1} \mathrm{H} \mathrm{NMR}\left(400 \mathrm{MHz}, \mathrm{CDCl}_{3}, \delta, \mathrm{ppm}\right): 1.95\left(\mathrm{~s}, 6 \mathrm{H}, 2 \mathrm{CH}_{3}\right)$, $4.65\left(\mathrm{~s}, 4 \mathrm{H}\right.$, benzylamine- $\left.\mathrm{CH}_{2}\right), 6.52(\mathrm{~s}, 1 \mathrm{H}, \mathrm{C}=\mathrm{CH}), 6.55(\mathrm{~d}, J=8.64 \mathrm{~Hz}, 2 \mathrm{H}$, aminophenyl- $H), 6.91$ (s, 2H, benzene-fused- $H$ ), 7.13-7.27 (m, $10 \mathrm{H}$, benzylamine- $H), 7.31(\mathrm{~d}, J=8.64 \mathrm{~Hz}, 2 \mathrm{H}$, aminophenyl- $H$ ). MS (NOBA, FAB > 0): $730[\mathrm{M}+\mathrm{H}]^{+}$. Anal. calcd. for $\mathrm{C}_{36} \mathrm{H}_{27} \mathrm{~S}_{8} \mathrm{~N}: \mathrm{C}, 59.22 ; \mathrm{H}, 3.72$; S, 35.13; found: C, 59.07; H, 3.58; S, 35.32.

p-Dibenzylaminophenyl benzene-fused bis tetrathiafulvalene 12c: Yellow powder. Yield: 93\%. M.p.: $213{ }^{\circ} \mathrm{C}$. TLC: $\mathrm{Rf}=0.66\left(\mathrm{CH}_{2} \mathrm{Cl}_{2}\right) .{ }^{1} \mathrm{H} \mathrm{NMR}\left(400 \mathrm{MHz}, \mathrm{CDCl}_{3}, \delta, \mathrm{ppm}\right): 4.66(\mathrm{~s}, 4 \mathrm{H}$, benzylamine- $\left.\mathrm{CH}_{2}\right), 6.53(\mathrm{~s}, 1 \mathrm{H}, \mathrm{C}=\mathrm{CH}), 6.56(\mathrm{~d}, J=8.68 \mathrm{~Hz}, 2 \mathrm{H}$, aminophenyl- $H), 6.93(\mathrm{~s}, 2 \mathrm{H}$, benzene-fused- $H), 7.14-7.25(\mathrm{~m}, 14 \mathrm{H}$, benzylamine- $H$, benzene- $H), 7.35(\mathrm{~d}, J=8.68 \mathrm{~Hz}, 2 \mathrm{H}$, 
aminophenyl- $H$ ). MS (NOBA, FAB > 0): $752[\mathrm{M}+\mathrm{H}]^{+}$. Anal. calcd. for $\mathrm{C}_{38} \mathrm{H}_{25} \mathrm{~S}{ }_{8} \mathrm{~N}: \mathrm{C}, 60.68 ; \mathrm{H}, 3.35$; S, 34.10; found: $\mathrm{C}, 60.56 ; \mathrm{H}, 3.25 ; \mathrm{S}, 34.29$.

p-Dibenzylaminophenyl benzene-fused bis tetrathiafulvalene 12d: Gold powder. Yield: 85\%. M.p.: $218{ }^{\circ} \mathrm{C}$. TLC: $\mathrm{Rf}=0.70\left(\mathrm{CH}_{2} \mathrm{Cl}_{2}\right) .{ }^{1} \mathrm{H}$ NMR $\left(400 \mathrm{MHz}, \mathrm{CDCl}_{3}, \delta, \mathrm{ppm}\right): 2.45\left(\mathrm{q}, J=6.9 \mathrm{~Hz}, 2 \mathrm{H}, \mathrm{CH}_{2}\right)$, $2.56\left(\mathrm{t}, J=6.9 \mathrm{~Hz}, 4 \mathrm{H}, 2 \mathrm{CH}_{2}\right), 4.65\left(\mathrm{~s}, 4 \mathrm{H}\right.$, benzylamine- $\left.\mathrm{CH}_{2}\right), 6.52(\mathrm{~s}, 1 \mathrm{H}, \mathrm{C}=\mathrm{CH}), 6.55(\mathrm{~d}, J=8.65 \mathrm{~Hz}$, $2 \mathrm{H}$, aminophenyl- $H), 6.91(\mathrm{~s}, 2 \mathrm{H}$, benzene-fused- $H), 7.13-7.28(\mathrm{~m}, 10 \mathrm{H}$, benzylamine- $H), 7.31(\mathrm{~d}$, $J=8.65 \mathrm{~Hz}, 2 \mathrm{H}$, aminophenyl- $H$ ). MS (NOBA, FAB $>0$ ): $742[\mathrm{M}+\mathrm{H}]^{+}$. Anal. calcd. for $\mathrm{C}_{37} \mathrm{H}_{27} \mathrm{~S}_{8} \mathrm{~N}$ : C, 59.88; H, 3.66; S, 34.56; found: C, 60.13; H, 3.86; S, 34.26.

\section{Conclusions}

We herein describe the synthesis and the characterization of novel unsymmetrically benzene-fused bis tetrathiafulvalenes bearing alkyl chains at one end of the $\pi$-electron rich unit and different functional groups $p$-nitrophenyl, $p$-aminophenyl or $p$-dibenzylaminophenyl at the other extreme. Different routes and reaction conditions were explored to form these compounds.

The synthetic method requires the preparation of three new precursors the 4-( $p$-nitrophenyl)-1,3dithiole-2-ylidenepiperidinium hexafluorophosphate 2, 4-( $p$-nitrophenyl)-1,3-dithiole-2-selenone 3 and the 2-(4-( $p$-nitrophenyl)-1,3-dithiole-2-ylidene)-1,3,5,7-tetrathia-s-indacene-6-one 5.

The electrochemical behavior of all donors was determined by cyclic voltammetry. Charge transfer complexes of the donors with TCNQ were prepared and the electrical conductivity of these materials was measured. Series of $p$-nitrophenyl benzene-fused bis tetrathiafulvalenes and $p$-aminophenyl benzene-fused bis tetrathiafulvalenes derivatives are conductors while series of $p$-dibenzylaminophenyl benzene-fused bis tetrathiafulvalenes are semi-conductors.

\section{Acknowledgments}

This work was partially supported by Algerian Research Ministry, MERS.

\section{Conflicts of Interest}

The authors declare no conflict of interest.

\section{References}

1. Canevet, D.; Sallé, M.; Zhang, G.; Zhang, D.; Zhu, D. Tetrathiafulvalene (TTF) derivatives: Key building-blocks for switchable processes. Chem. Commun. 2009, 17, 2245-2269.

2. Fourmigue, M.; Batail, P. Activation of hydrogen- and halogen-bonding interactions in tetrathiafulvalene-based crystalline molecular conductors. Chem. Rev. 2004, 104, 5379-5418.

3. Singleton, J. Why do physicists love charge-transfer salts? J. Solid State Chem. 2002, 168, 675-689.

4. Bouguessa, S.; Gouasmia, A.K.; Golhen, S.; Ouahab, L.; Fabre, J.M. Synthesis and characterization of TTF-type precursors for the construction of conducting and magnetic molecular materials. Tetrahedron Lett. 2003, 44, 9275-9278.

5. Dressel, M.; Drichko, N. Optical properties of two-dimensional organic conductors: Signatures of charge ordering and correlation effects. Chem. Rev. 2004, 104, 5689-5716. 
6. Jérome, D. Organic conductors: From charge density wave TTF-TCNQ to superconducting (TMTSF) ${ }_{2} \mathrm{PF}_{6}$. Chem. Rev. 2004, 104, 5565-5592.

7. Liu, L.H.; Zhang, H.; Li, A.F.; Xie, J.W.; Jiang, Y.B. Intramolecular charge transfer dual fluorescent sensors from 4-(dialkylamino)benzanilides with metal binding site within electron acceptor. Tetrahedron 2006, 62, 10441-10449.

8. Balandier, J.Y.; Belyasmine, A.; Salle, M. Tetrathiafulvalene-imine-pyridine assemblies for $\mathrm{Pb}^{2+}$ recognition. Eur. J. Org. Chem. 2008, 2, 269-276.

9. Goze, C.; Dupont, N.; Beitler, E.; Leiggener, C.; Jia, H.; Monbaron, P. Ruthenium(II) coordination chemistry of a fused donor-acceptor ligand: Synthesis, characterization, and photoinduced electron-transfer reactions of $\left[\left\{\mathrm{Ru}(\mathrm{bpy})_{2}\right\}_{\mathrm{n}}(\mathrm{TTF}-\mathrm{ppb})\right]\left(\mathrm{PF}_{6}\right)_{2 \mathrm{n}}(n=1,2)$. Inorg. Chem. 2008, 47, 11010-11017.

10. Martin, N.; Sánchez, L.; Herranz, M.Á.; Illescas, B.; Guldi, D.M. Electronic communication in tetrathiafulvalene $(\mathrm{TTF}) / \mathrm{C}_{60}$ systems: Toward molecular solar energy conversion materials? Acc. Chem. Res. 2007, 40, 1015-1024.

11. Sommer, M.; Huettner, S.; Thelakkat, M. Donor-acceptor block copolymers for photovoltaic applications. J. Mater. Chem. 2010, 20, 10788-10797.

12. Li, Y.; Xue, L.; Li, H.; Li, Z.; Xu, B.; Wen, S. Energy level and molecular structure engineering of conjugated donor-acceptor copolymers for photovoltaic applications. Macromolecules 2009, 42, 4491-4499.

13. Chen, W.; Cava, M.P.; Takassi, M.A.; Metzger, R.M. Synthesis of bis(2,5-dimethylpyrrolo[3,4d])-tetrathiafulvalene, an annelated TTF derivative with good electron donor properties. J. Am. Chem. Soc. 1988, 110, 7903-7904.

14. Murata, T.; Morita, Y.; Fukui, K.; Sato, K.; Shiomi, D.; Takui, T.; Maesato, M.; Yamochi, H.; Saito, G.; Nakasuji, K. A purely organic molecular metal based on a hydrogen-bonded charge-transfer complex: Crystal structure and electronic properties of TTF-imidazole-p-chloranil. Angew. Chem. Int. Ed. 2004, 43, 6343-6346.

15. Murata, T.; Morita, Y.; Yakiyama, Y.; Fukui, K.; Yamochi, H.; Saito, G.; Nakasuji, K. Hydrogen-bond interaction in organic conductors: Redox activation, molecular recognition, structural regulation, and proton transfer in donor-acceptor charge-transfer complexes of TTF-imidazole. J. Am. Chem. Soc. 2007, 129, 10837-10846.

16. Zhou, Y.; Zhang, D.; Zhu, L.; Shuai, Z.; Zhu, D. Binaphthalene molecules with tetrathiafulvalene units: CD spectrum modulation and new chiral molecular switches by reversible oxidation and reduction of tetrathiafulvalene units. J. Org. Chem. 2006, 71, 2123-2130.

17. Delogu, G.; Fabbri, D.; Dettori, M.A.; Sallé, M.; le Derf, F.; Blesa, M.J.; Allain, M. Electroactive $\mathrm{C}_{2}$ symmetry receptors based on the biphenyl scaffold and tetrathiafulvalene units. J. Org. Chem. 2006, 71, 9096-9103.

18. Bryce, M.R.; Devonport, W.; Goldberg, L.M.; Wang, C. Macromolecular tetrathiafulvalene chemistry. Chem. Commun. 1998, 9, 945-951.

19. Christensen, C.A.; Goldenberg, L.M.; Bryce, M.R.; Becher, J. Synthesis and electrochemistry of a tetrathiafulvalene $(\mathrm{TTF})_{21}$-glycol dendrimer: Intradendrimer aggregation of TTF cation radicals. Chem. Commun. 1998, 4, 509-510. 
20. Metzger, R.M.; Chen, B.; Höpfner, U.; Lakshmikantham, M.V.; Vuillaume, D.; Kawai, T.; Wu, X.; Tachibana, H.; Hughes, T.V.; Sakurai, H.; et al. Unimolecular electrical rectification in hexadecylquinolinium tricyanoquinodimethanide. J. Am. Chem. Soc. 1997, 119, 10455-10466.

21. Iimori, T.; Naito, T.; Ohta, N. Unprecedented optoelectronic function in organic conductor: Memory effect of photoswitching controlled by voltage pulse width. J. Phys. Chem. C 2009, 113, 4654-4661.

22. Kim, H.; Goddard, W.A.; Jang, S.S.; Dichtel, W.R.; Heath, J.R.; Stoddart, J.F. Free energy barrier for molecular motions in bistable [2] rotaxane molecular electronic devices. J. Phys. Chem. A 2009, 113, 2136-2143.

23. Bendikov, M.; Wudl, F.; Perepichka, D.F. Tetrathiafulvalenes, oligoacenenes, and their buckminsterfullerene derivatives: The brick and mortar of organic electronics. Chem. Rev. 2004, 104, 4891-4945.

24. Segura, J.L.; Priego, E.M.; Martin, N. New functionalized and soluble bis-tetrathiafulvalene derivatives as building blocks in the construction of fullerene-derived electroactive triads. Tetrahedron Lett. 2000, 41, 7737-7741.

25. Gautier, N.; Cariou, M.; Gorgues, A.; Hudhomme, P. A novel array in extended tetrathiafulvalenes (TTF): The "H" shape. Tetrahedron Lett. 2000, 41, 2091-2095.

26. Diaz, M.C.; Illescas, B.M.; Martin, N.; Viruela, R.; Viruela, P.M.; Orti, E.; Brede, O.; Zilbermann, I.; Guldi, D.M. Highly conjugated $p$-quinonoid $p$-extended tetrathiafulvalene derivatives: A class of highly distorted electron donors. Chem. Eur. J. 2004, 10, 2067-2077.

27. Santos, J.; Illescas, B.M.; Martin, N.; Adrio, J.; Carretero, J.C.; Viruela, R.; Orti, E.; Spänig, F.; Guldi, D.M. A fully conjugated TTF- $\pi$-TCAQ system: Synthesis, structure, and electronic properties. Chem. Eur. J. 2011, 17, 2957-2964.

28. Gao, X.; Wu, W.; Liu, Y.; Qiu, W.; Sun, X.; Yu, G.; Zhu, D. A facile synthesis of linear benzene-fused bis (tetrathiafulvalene) compounds and their application for organic field-effect transistors. Chem. Commun. 2006, 26, 2750-2752.

29. Abbaz, T.; Bendjeddou, A.; Gouasmia, A.K.; Regainia, Z.; Villemin, D. Synthesis and electrochemical proprieties of novel unsymmetrical bis-tetrathiafulvalenes and electrical conductivity of their charge transfer complexes with tetracyanoquinodimethane (TCNQ). Int. J. Mol. Sci. 2012, $13,7872-7885$.

30. Abbaz, T.; Gouasmia, A.K.; Fujiwara, H.; Hiraoka, T.; Sugimoto, T.; Taillefer, M.; Fabre, J.M. New TTF and bis-TTF containing thiophene units: Electrical properties of the resulting salts. Synth. Met. 2007, 157, 508-516.

31. Boudiba, L.; Gouasmia, A.K.; Golhen, S.; Ouahab, L. Synthesis and X-ray crystal structures of radical cation salts of benzo-TTF derivatives with $\mathrm{Cu}_{2} \mathrm{Cl}_{6}{ }^{2-}, \mathrm{CuCl}_{4}{ }^{2-}$ and $\mathrm{ClO}_{4}{ }^{-}$anions. Synth. Met. 2011, 161, 1800-1804.

32. Bouguessa, S.; Gouasmia A.K.; Ouahab, L.; Golhen, S.; Fabre, J.M. Preparations and characterizations of new series of TTF ligands containing a nitrogen aromatic heterocycle. Synth. Met. 2010, 160, 361-367.

33. Abbaz, T.; Bendjeddou, A.; Gouasmia, A.k.; Bouchouk, D.; Boualleg, C.; Kaouachi, N.; Inguimbert, N.; Villemin, D. Synthesis, characterization and antibacterial activity of cyclic sulfamide linked to tetrathiafulvalene (TTF). Lett. Org. Chem. 2014, 11, 59-63. 
34. Kaboub, L.; Slimane, F.; Gouasmia, A.K. Synthesis and properties of novel unsymmetrical donor molecules containing $p$-acetoxy- or $p$-hydroxyphenyl units. Molecules 2006, 11, 776-785.

35. Misaki, Y.; Nishikawa, H.; Kawakami, K.; Yamabe, T.; Mori, T.; Inokuchi, H.; Mori, H.; Tanaka, S. Ethylenedioxy substituted 2,5-bis(1',3'-dithiol-2'-ylidene)-1,3,4,6-tetrathiapentalenes and their conducting salts. Chem. Lett. 1993, 22, 2073-2076.

36. Otsubo, T.; Shiomi, Y.; Imamura, M.; Kittaka, R.; Ohnishi, A.; Tagawa, H.; Aso, Y.; Ogura, F. Syntheses and properties of derivatives of 2-(thiopyran-4-ylidene)-1,3-dithiole and selenium analogues as novel unsymmetrical electron donors. J. Chem. Soc. Perkin Trans. 2 1993, 10, $1815-1824$.

37. Neiland, O.Y.; Balodis, K.A.; Khodorkovskii, V.Y.; Tilika, V.Z. Synthesis of tetrathiafulvalene derivatives by dimerization of 1,3-dithiolselenones-2 using triphenylphosphine. Chem. Heterocycl. Compd. 1991, 9, 1278-1279.

38. Cava, M.P.; Lakshmikantham, M.V. Unsymmetrical tetrathiafulvalenes. Ann. N. Y. Acad. Sci. 1978, 313, 355-360.

39. Spencer, H.K.; Cava, M.P.; Garito, A.F. Organic metals: Synthesis of benzotetrathiafulvalene. J. Chem. Soc. Chem. Commun. 1976, 23, 966-967.

40. Fabre, J.M. Dimensionality and electrical properties in organic synthetic metals-current results through selected recent examples. J. Solid State Chem. 2002, 168, 367-383.

41. Segura, J.L.; Martin, N. New concepts in tetrathiafulvalene chemistry. Angew. Chem. Int. Ed. 2001, 40, 1372-1409.

42. Frère, P.; Skabara, P. Salts of extended tetrathiafulvalene analogues: Relationships between molecular structure, electrochemical properties and solid state organisation. J. Chem. Soc. Rev. 2005, 34, 69-68.

43. Frisch, M.J.; Trucks, G.W.; Schlegel, H.B.; Scuseria, G.E.; Robb, M.A.; Cheeseman, J.R.; Scalmani, G.; Barone, V.; Mennucci, B.; Petersson, G.A.; et al. Gaussian 09, Revision C.01; Gaussian Inc.: Wallingford, CT, USA, 2010.

44. Hehre, W.J.; Ditchfield, R.; Pople, J.A. Self-consistent molecular orbital methods. XII. Further extensions of Gaussian-type basis sets for use in molecular orbital studies of organic molecules. J. Chem. Phys. 1972, 56, 2257-2261.

45. Becke, A.D. Density-functional exchange-energy approximation with correct asymptotic behavior. Phys. Rev. A 1988, 38, 3098-3100.

46. Becke, A.D. Density-functional thermochemistry. III. The role of exact exchange. J. Chem. Phys. 1993, 98, 5648-5652.

47. Lee, C.; Yang, W.; Parr, R.G. Development of the Colle-Salvetti correlation-energy formula into a functional of the electron density. Phys. Rev. B 1998, 37, 785-789.

(C) 2014 by the authors; licensee MDPI, Basel, Switzerland. This article is an open access article distributed under the terms and conditions of the Creative Commons Attribution license (http://creativecommons.org/licenses/by/3.0/). 